\title{
African green monkey Zika models
}

Haddow, A.D. et al. PLoS Neglect Trop D (2020). https://doi.org/10.1371/journal.pntd.0008107

Interest in otherwise neglected tropical diseases often follows their outbreak cycles. A few years ago, attention was on Zika virus, a flavivirus that causes relatively mild or asymptomatic infections in adults but that can lead to severe congenital birth defects in children born to infected mothers.

The 2015/2016 New World outbreak may have subsided, but the virus is not gone. Given its varied ecological niches and the ease by which Zika can be transmitted by mosquitoes, as well as evidence for sexual transmission and an ability to spread over long distances via air travel, epidemic potential remains. "There are lots of red flags with this virus," says virologist Andrew Haddow, a contractor with General Dynamics Health Solutions working at the US Army Medical Research Institute of Infectious Diseases and a member of the Global Virus Network Zika Virus Task Force.

Asian strains were behind the recent spate of infections in tropical regions of the Americas, but the virus most likely originated in Africa. There it continues to circulate, though outbreaks and incidences of birth defects may go undiagnosed or underreported. As other labs focused on Asian strains, Haddow and his team have been studying the African lineage, in cell lines, rodents, and nonhuman primates, including rhesus and cynomolgus macaques and most recently, African green monkeys (AGMs).

Macaques, with Southeast Asian origins, would only recently have been exposed to Zika virus; AGMs, meanwhile, live in regions where the virus has long circulated, which the team speculated might make them more sensitive to infection. In a pilot study, Haddow and his team inoculated male and female AGMs three ways: subcutaneously, to model the virus's primary mode of mosquito-borne transmission, and intravaginally or intrarectally to model secondary sexual transmission.

Overall the AGMs appeared healthy, but in a battery of tests the team observed swollen lymph nodes in some of the animals' abdomens via ultrasound, as well as signs of acute liver injury. Viremia and antibody response was observed in different animals, as was viral shedding. One female in particular shed high levels of the virus from Day 5 post-inoculation to Day 21; this suggests super shedders might be super spreaders, with a role in moving the virus between infected and naive populations.

Two birds, one stone, says Haddow. As natural hosts, AGMs could offer clues to enzootic amplification cycles of Zika virus. With infection dynamics similar to those reported in humans, they can also serve as an alternative nonhuman primate model to macaques for studying transmission and disease progression and for testing candidate vaccines and antivirals that are, even as headlines have died down, making their way towards the clinic.

\section{Ellen Neff}

Published online: 20 July 2020

https://doi.org/10.1038/s41684-020-0615-0

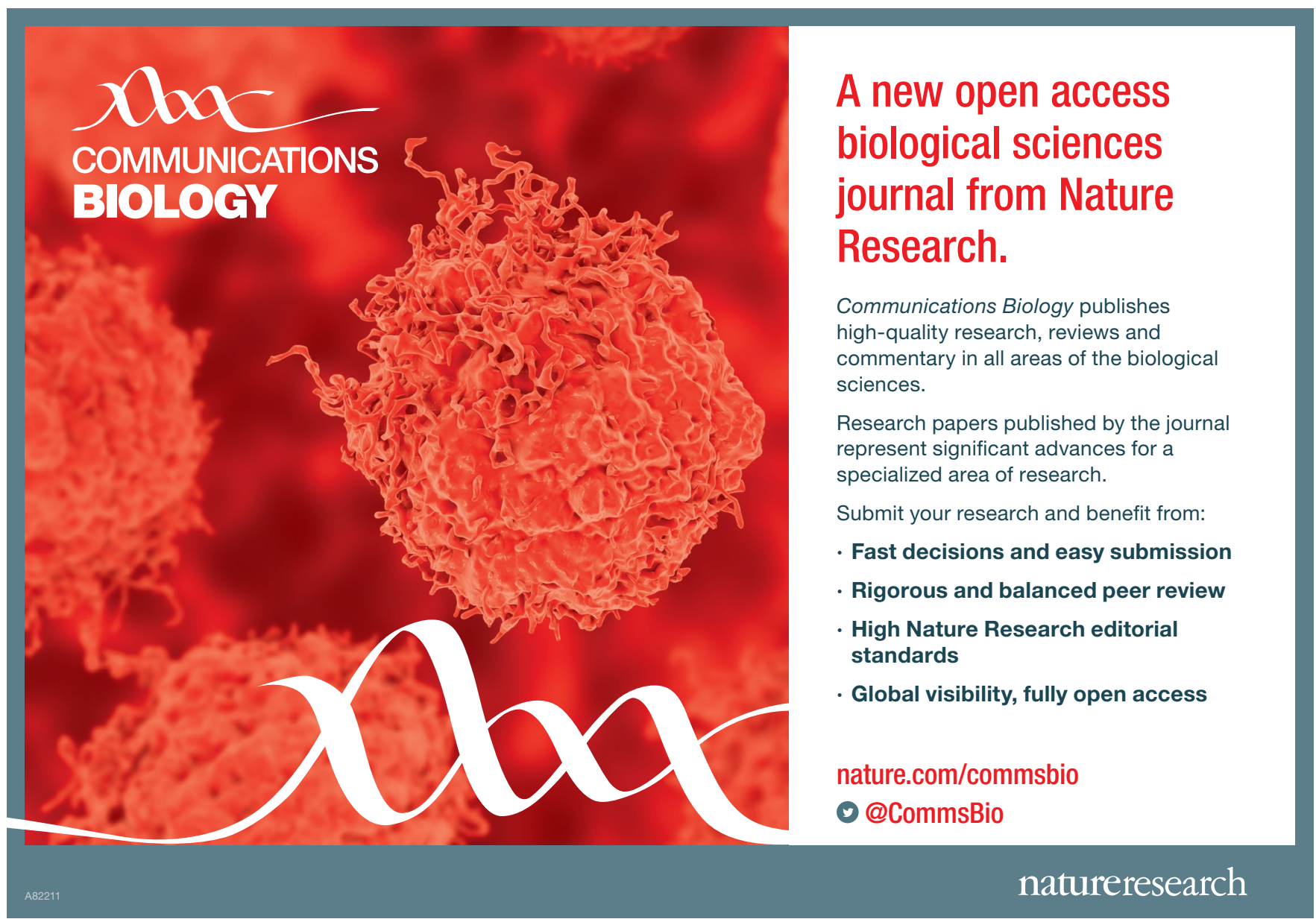

